\title{
Über eine pulmonale Infektion durch Mycobacterium malmoense
}

\section{W. Handrick ${ }^{1}$ \\ I. Schwede ${ }^{2}$ \\ O. Ebeling ${ }^{3}$ \\ B. Wogawa ${ }^{4}$ \\ F. Berthold ${ }^{2}$}

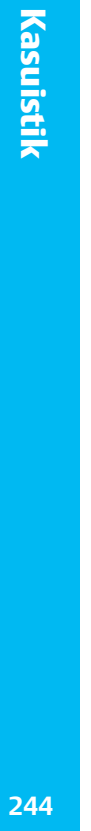

Pulmonary Infection due to Mycobacterium Malmoense

\section{Zusammenfassung}

Bei einem 49-jährigen Patienten mit destruierenden Lungenveränderungen wurden im Sputum Mykobakterien nachgewiesen, die weitere Differenzierung ergab M. malmoense. Die zunächst begonnene antituberkulöse Therapie wurde beendet und eine Behandlung mit Ethambutol, Rifabutin, Clarithromycin und Ciprofloxacin begonnen.

Dieser Therapie folgte eine Langzeitgabe von Azithromycin bzw. Clarithromycin. Eine Eradikation der Erreger aus dem Respirationstrakt gelang bisher nicht (ungenügende Compliance, Therapiepausen infolge Nebenwirkungen, fortbestehender Nikotinabusus).

Infektionen durch M. malmoense sind selten. Sie betreffen meist Patienten mit disponierenden Grundkrankheiten. In den meisten Fällen handelt es sich um pulmonale Infektionen.

Die bisher am häufigsten in der Therapie eingesetzten Antibiotika sind Rifampicin bzw. Rifabutin, Ethambutol und Clarithromycin.

\section{Abstract}

M. malmoense could be cultivated in sputum samples of a 49-year-old patient with destructive pulmonary disease. The conventional antituberculous therapy (started because initially a presumptive diagnosis of tuberculosis was established) was altered to ethambutol, rifabutin, clarithromycin and ciprofloxacin, followed by a long-time therapy with azithromycin or clarithromycin. But till now it was not possible to eradicate the mycobacteria from the respiratory tract (insufficient compliance, interruptions of the therapy due to side effects, excessive smoking).

Infections due to M. malmoense are rare events. Many patients have disposing underlying diseases. In most cases it is a pulmonary infection.

The most frequent used antibiotics are rifampicin (or rifabutin), ethambutol and clarithromycin.
Nicht-tuberkulöse Mykobakterien gewinnen als Infektionserreger beim Menschen zunehmend an Bedeutung.

Neben Mycobacterium (M.) avium-intracellulare ist hier vor allem M. malmoense zu nennen.
Um auf diese Problematik hinzuweisen, möchten wir im Folgenden über einen Patienten mit einer chronischen pulmonalen Infektion durch M. malmoense berichten.

Institutsangaben

${ }^{1}$ Institut für Medizinische Diagnostik, Berlin

${ }^{2}$ Institut für Medizinische Diagnostik Oderland, Frankfurt (Oder)

${ }^{3}$ Humaine Klinikum Bad Saarow, Klinik für Innere Medizin

${ }^{4}$ Humaine Klinikum Bad Saarow, Zentrallabor

Korrespondenzadresse

Prof. Dr. W. Handrick · Institut für Medizinische Diagnostik Berlin · Dr. Schmidt \& Kollegen · Nicolaistraße 22 . 12247 Berlin·E-mail: Prof.W.Handrick@imd-Berlin.de

Eingang: 4. Juni 2004 - Nach Revision alkzeptiert: 23. Februar 2005

Bibliografie

Pneumologie 2005; 59: 244-247 @ Georg Thieme Verlag KG Stuttgart • New York

DOI 10.1055/s-2004-830243

ISSN 0934-8387 
Kasuistik

Der 49-jährige Patient mit chronisch-obstruktiver Bronchitis (bei langjährigem Nikotinabusus) befand sich seit Februar/März 1998 wegen rezidivierender Infekte in ambulanter Behandlung.

Die bildgebende Diagnostik zeigte destruierende, teils fibrozirrhotische, teils kavernöse Lungenveränderungen.

Im Sputum vom 10.6.1998 wurden erstmalig mikroskopisch säurefeste Stäbchen nachgewiesen, die sich auch kulturell bestätigten. Nachfolgende Materialeinsendungen (Sputum und Bronchialsekret) ergaben ebenfalls säurefeste Stäbchen bzw. in der Kultur Mykobakterien.

Noch bevor das endgültige Differenzierungsergebnis vorlag und obwohl der Tuberkulintest (10 E GT) mit 4 mm negativ war, wurde in Anbetracht der klinischen Symptomatik mit einer antituberkulösen Therapie mit Isoniazid (INH), Rifampicin, Pyrazinamid und Ethambutol begonnen.

Ab 1. 7.1998 waren die mikroskopischen und (retrospektiv) kulturellen Kontrollen negativ, so dass der Patient am 10. 7.1998 bei subjektivem Wohlbefinden in die ambulante Betreuung entlassen werden konnte. Es wurde empfohlen, ab August 1998 eine Umstellung auf eine Zweifachkombination mit Rifampicin und INH für weitere Monate vorzunehmen, vorbehaltlich des Ergebnisses der endgültigen Differenzierung der Erreger.

Der angezüchtete Mykobakterien-Stamm konnte im Referenzlabor schließlich als M. malmoense differenziert werden'.

In der Folgezeit wechselte der Patient mehrfach den Arzt bzw. blieb ohne ärztliche Betreuung, so dass die Therapie weder korrigiert noch fortgesetzt werden konnte.

${ }^{1}$ Für die Differenzierung danken wir den Mitarbeitern des Nationalen Referenzzentrums für Mykobakterien im Forschungszentrum Borstel.

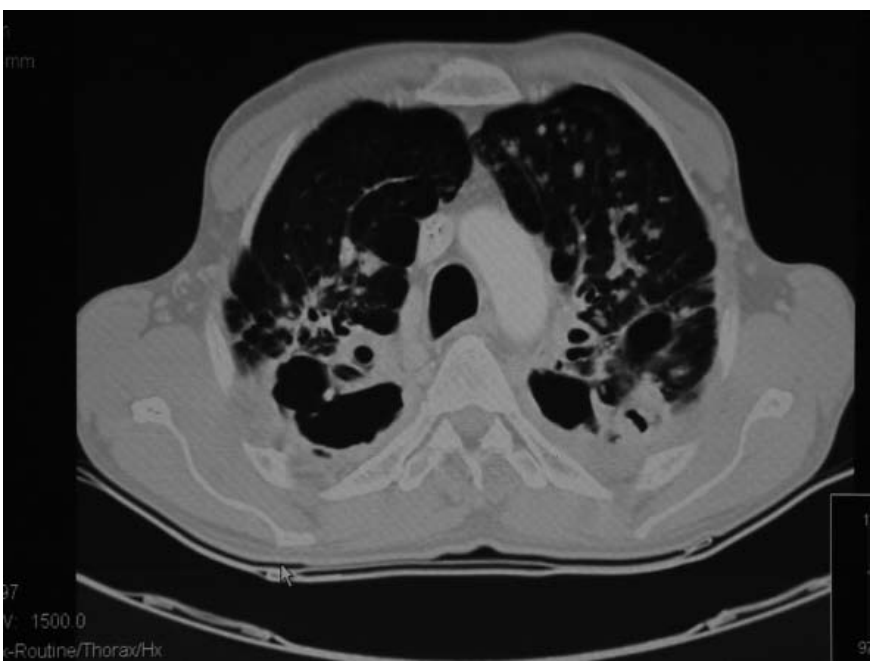

Abb. 1 CT-Schnitt der Lunge zeigt infiltrativ-kavernöse OberlappenDestruktion beidseits mit Pleuraverdickung.
Erst am 28. 5. 2003 erfolgte bei Hämoptoe über den Notarzt eine weitere stationäre Aufnahme, nachdem schon über ein halbes Jahr Reizhusten, Dyspnoe und Gewichtsabnahme bestanden hatten.

Außer der Röntgenuntersuchung der Lunge erfolgten ein ThoraxCT mit Kontrastmittel sowie eine Bronchoskopie (Blennorrhoe aus beiden Oberlappen, schleimiges Sekret aus S 6 bds., Freispülen der Segmentostien).

Im Sputum fanden sich mikroskopisch säurefeste Stäbchen.

Trotz der Tatsache, dass bei diesem Patienten 1998 eine M. malmoense-Infektion diagnostiziert worden war, stellte man jetzt die Verdachtsdiagnose „Lungentuberkulose“ und begann wieder eine Behandlung mit oben genannter Vierfachkombination (eine PCR wurde leider nicht durchgeführt). Die Differenzierung des angezüchteten Mykobakterien-Stammes ergab aber wieder M. malmoense ${ }^{1}$ (sensibel gegenüber Ethambutol, Ofloxacin, Clarithromycin, resistent gegenüber INH, Rifampicin, Pyrazinamid und Streptomycin).

Die Behandlung wurde mit Ethambutol, Rifabutin, Ciprofloxacin und Clarithromycin fortgesetzt. Der Patient rauchte weiterhin täglich ca. 20 Zigaretten.

Das in dieser Zeit durchgeführte Lungen-CT zeigte einen im Wesentlichen unveränderten Lungenbefund (Abb. 1 und Abb. 2).

Wegen zunehmender gastrointestinaler Beschwerden, die am ehesten auf die genannte Therapie zurückzuführen waren, wurde die Behandlung ab März 2004 lediglich mit Azithromycin fortgeführt (zeitweilig wurde der Patient parenteral ernährt). Aber auch dieses Mittel wurde nur schlecht toleriert, so dass intermittierend Therapiepausen erfolgten.

Zunächst ergaben die mikrobiologischen Sputumkontrollen keine Nachweise von Mykobakterien, im September 2004 konnte aber während eines stationären Aufenthaltes (gastrointestinale

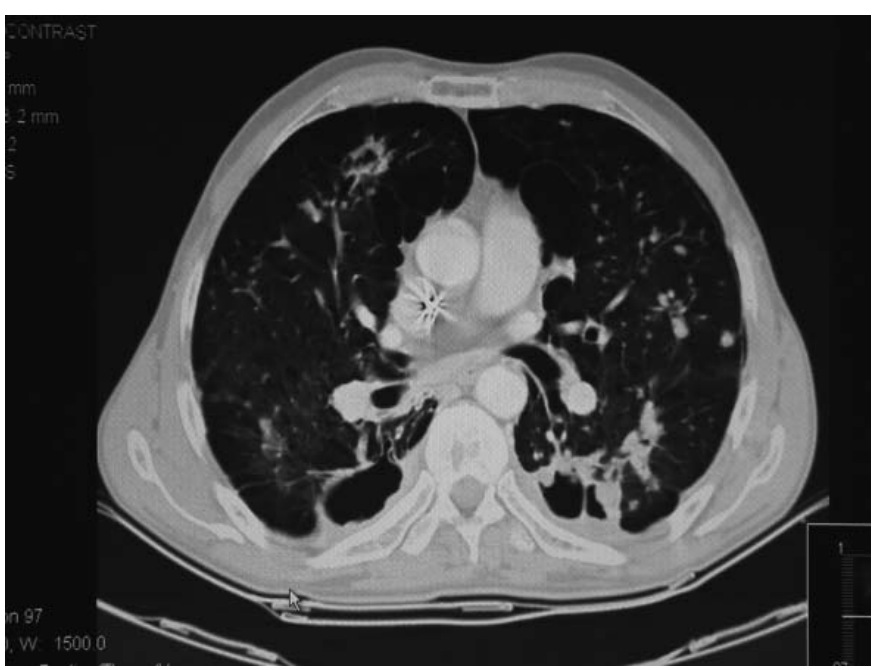

Abb. 2 CT-Schnitt der Lunge zeigt Infiltrate in beiden Oberlappen, Bullae mit Bezug zur Pleura mediastinalis, Kavernen mit pleuraler Verschwartung in $\mathrm{S} 6$ beidseits. 
Beschwerden, verschlechterter Allgemeinzustand) erneut $\mathrm{M}$. malmoense aus dem Bronchialsekret angezüchtet werden.

Es erfolgte eine Behandlung mit Ciprofloxacin und Clarithromycin i.v. Unter dieser Therapie besserte sich der Zustand des Patienten deutlich.

Nach Entlassung wurde die Therapie mit Clarithromycin p.o. und Ethambutol fortgesetzt. Eine erneute parenterale AntibiotikaTherapie ist geplant.

\section{Diskussion}

Gute epidemiologische Daten zum Vorkommen von Infektionen durch M. malmoense liegen nicht vor, seit 1980 wird aber zunehmend über solche Infektionen berichtet [22].

Überwiegend handelt es sich um Erwachsene, Kinder erkranken seltener $[4,8-10]$. In den publizierten Fallserien findet sich meist ein Überwiegen des männlichen Geschlechts.

M.-malmoense-Infektionen treten weltweit auf $[1,4]$, die meisten Fälle wurden aber bisher in Nordeuropa diagnostiziert, z. B. in Großbritannien $[5,13,14,20,21]$, Irland [18], Schweden $[7,10,11]$, aber auch aus Deutschland $[8,15,16]$, der Schweiz $[3,9,22]$, Frankreich [6] und Italien [19] liegen inzwischen Publikationen vor.

Invasive bzw. disseminierte M.-malmoense-Infektionen betreffen meist Patienten mit bestimmten Grundkrankheiten, z. B. solche mit immunsuppressiver Therapie [5,15,21], AIDS [22], Diabetes mellitus [3,15], malignen Erkrankungen [6,7], Alkoholbzw. Nikotinabusus $[1,3,4,10,20]$ sowie chronischen Lungenerkrankungen [22].

Die Erreger stammen wahrscheinlich aus der Umwelt (Boden, Wasser).

Die klinische Symptomatik wird bestimmt vom betroffenen Organ und dem Schweregrad der Erkrankung. Bei den Erwachsenen handelt es sich in den meisten Fällen um pulmonale Infektionen [10]. Diese unterscheiden sich klinisch kaum von Lungen-Tuberkulosen [3, 8]. Wie bei der Tuberkulose können sich auch hier Kavernen entwickeln $[3,14]$, aber auch eine bronchoösophageale Fistel wurde beobachtet [15].

Eine eher chronische Symptomatik ist auch typisch, wenn sich die Infektion in einem anderen Organsystem abspielt, z. B. im Bereich der Haut [6,17], eines Gelenks bzw. Knochens [5,21] oder als Tenosynovitis verläuft $[12,18,22]$.

Bei deutlich beeinträchtigter Abwehrlage kommt es auch zu disseminierten Infektionen [7,22].

Bei immunkompetenten Kindern kann M. malmoense (selten) eine zervikale Lymphadenitis auslösen $[4,8$-10,16,22].

Der Tuberkulin-Test ist üblicherweise negativ oder schwach positiv. Die wichtigsten Untersuchungsmaterialien zum Erreger- nachweis sind Sputum, BAL-Flüssigkeit, Trachealsekret und Punktate bzw. Biopsate. Bei disseminierten Infektionen gelang der Erregernachweis auch aus Stuhl bzw. Abszesseiter [22].

Die Anzucht dieser nicht-tuberkulösen Mykobakterien gelingt auf Nährböden, die zur Tuberkulose-Diagnostik geeignet sind. M. malmoense wächst langsam (besser bei $30^{\circ} \mathrm{C}$ ), es wird daher eine Inkubationszeit von $8-12$ Wochen empfohlen $[3,8,22]$. Die endgültige Identifizierung sollte in Speziallaboratorien erfolgen.

Der einmalige Nachweis von M. malmoense im Sputum beweist nicht das Vorliegen einer pulmonalen Infektion, es könnte sich in einem solchen Fall auch um eine Besiedlung handeln.

Für das Vorliegen einer mykobakteriellen Infektion spricht der mehrmalige Erregernachweis bzw. der Nachweis aus einem Aspirat bzw. Biopsat im Zusammenhang mit einer entsprechenden klinischen Symptomatik, aber auch der Nachweis einer granulomatösen Entzündung (evtl. mit Langhansschen Riesenzellen und Verkäsung) [4, 8,9,12,17].

Die American Thoracic Society hat diagnostische Kriterien definiert, die erfüllt sein müssen, bevor die Diagnose „Lungeninfektion durch nicht-tuberkulöse Mykobakterien“ gestellt werden kann [2].

Differenzialdiagnostisch wichtig ist vor allem die Unterscheidung zwischen M.-malmoense-Infektionen einerseits und Infektionen durch andere Mykobakterien andererseits (M. tuberculosis bzw. andere nicht-tuberkulöse Mykobakterien).

Nach Eintreffen des Befundes „säurefeste Stäbchen“ erfolgte in vielen Fällen zunächst eine antituberkulöse Therapie [8]. Erst wenn die angezüchteten Erreger als M. malmoense typisiert worden waren, wurde mit einer erregerspezifischen Therapie begonnen bzw. erfolgte eine Therapieumstellung.

Bei jedem angezüchteten Stamm sollte eine Resistenz-Testung durchgeführt werden. In den meisten Fällen besteht Resistenz gegenüber INH und Pyrazinamid $[8,12,16]$.

Mehrere Autoren wiesen aber darauf hin, dass die Ergebnisse der Resistenzbestimmung in vitro nicht unbedingt mit der klinischen Wirksamkeit korrelieren [14,22]. Durch In-vitro-Untersuchungen konnte gezeigt werden, dass es durch die Kombination mehrerer Mittel zu synergistischen Effekten kommt [11].

Die meisten Therapieerfahrungen liegen mit Rifampicin bzw. Rifabutin, Ethambutol und Clarithromycin vor [1,6, 8,15,21,22], so dass als First-line-Therapie am ehesten diese Dreierkombination empfohlen werden kann.

Aber auch Amikacin [7], Clofazimin [22], Prothionamid [15,16] und Ofloxacin [15] wurden eingesetzt.

Ein durch entsprechende Studien gesichertes optimales Therapieregime gibt es aber bisher nicht. Die Angaben zur Therapiedauer schwanken zwischen 9 und 24 Monaten, die Dauer wird offensichtlich bestimmt von der individuellen Situation des Patienten. 
Bei ungenügendem Ansprechen auf die Antibiotikatherapie bzw. bei Resistenz sind u.U. operative Maßnahmen (z.B. partielle Lungensektion) nötig [1,3].

Die Therapie der Wahl bei Lymphadenitis durch M. malmoense bei primär gesunden Kindern besteht in der vollständigen Exzision des betroffenen Lymphknotens [22].

Patienten mit M.-malmoense-Infektionen gelten (zumindest für immunologisch gesunde Kontaktpersonen) nicht als kontagiös, somit entfallen Isolierung der Patienten und Umgebungsuntersuchungen $[3,22]$.

Bei unserem Patienten sprechen die Anamnese (chronischer Nikotinabusus), die chronische Lungenerkrankung (COPD) als Dispositionsfaktor, der mehrmalige Nachweis von M. malmoense im Sputum bzw. Bronchialsekret (und negative Befunde bezüglich anderer potenzieller Verursacher dieser Veränderungen), und auch der negative Tuberkulintest dafür, dass es sich tatsächlich um eine M.-malmoense-Infektion handelt. Die oben genannten diagnostischen Kriterien [2] wurden erfüllt.

Der weitere Verlauf bei unserem Patienten zeigt, wie schwierig die Therapie solcher Infektionen im Einzelfall sein kann.

Eine sichere Eradikation der Erreger ist bis jetzt nicht gelungen. Hierbei dürften die bestehenden destruierenden Lungenveränderungen, der fortbestehende Nikotinabusus, die Nebenwirkungen der Antibiotika und die daraus folgende ungenügende Compliance des Patienten bzw. notwendig gewordene Therapiepausen eine Rolle spielen.

\section{Literatur}

${ }^{1}$ Al-Moamary MS, Black W, Elwood K. Pulmonary disease due to Mycobacterium malmoense in British Columbia. Can Respir J 1998; 5: $135-138$

${ }^{2}$ American Thoracic Society. Diagnosis and treatment of disease caused by nontuberculous mycobacteria. Am J Respir Crit Care Med 1997; 156: S1 - S25

3 Barandun J, Salfinger M, Gräni R et al. Diagnosis and treatment of pulmonary infections with Mycobacterium malmoense. Schweiz med Wschr 1991; 121: 1767-1172

${ }^{4}$ Buchholz UT, Mc Neil MM, Keyes LE et al. Mycobacterium malmoense infections in the United States, January 1993 through June 1995. Clin Infect Dis 1998; 27: $551-558$
${ }^{5}$ Callaghan R, Allen M. Mycobacterium malmoense infection of the knee. Ann Rheum Dis 2003; 62: 1047 - 1048

${ }^{6}$ Doutre MS et al. Cutaneous infection by Mycobacterium-malmoense in a patient with myelodysplastic syndrome. J Roy Soc Med 1993; 86: $110-111$

${ }^{7}$ Engervall P, Björkholm M, Petrini B et al. Disseminated Mycobacterium malmoense infection in a patient with chronic granulocytic leukemia. J Int Med 1993; 234: 231 - 233

${ }^{8}$ Enzensberger R, Hunfeld K-P, Krause M et al. Mycobacterium malmoense infections in immunocompetent patients. Eur J Clin Microbiol Infect Dis 1999; 18: 579-581

${ }^{9}$ Fabbri J, Welge-Lüssen A, Frei R et al. Zervikale Lymphadenitis mit Mycobacterium malmoense bei einem Kind: Fallbeschreibung und Differenzialdiagnose der zervikalen Lymphadenitis und Lymphadenopathien. Schweiz med Wschr 1993; 123: 1756-1761

${ }^{10}$ Henriques B, Hoffner SE, Petrini B et al. Infection with Mycobacterium malmoense in Sweden: report of 221 cases. Clin Infect Dis 1994; 18: $596-600$

${ }^{11}$ Hoffner SE, Hjelm U, Källenius G. Susceptibility of Mycobacterium malmoense to antibacterial drugs and drug combinations. Antimicrob Ag Chemother 1993; 37: 1285-1288

12 Osterwalder C, Salfinger M, Sulser H. Mycobacterium malmoense Infektionen der Beugesehnenscheiden. Handchir Mikrochir Plast Chir 1992; $24: 210-214$

${ }^{13}$ Research Committee of the British Thoracic Society. First randomised trial of treatments for pulmonary disease caused by M. avium intracellulare, M. malmoense, and M. xenopi in HIV negative patients: rifampicin, ethambutol and isoniazid versus rifampicin and ethambutol. Thorax 2001; 56: $167-172$

${ }^{14}$ Research Committee of the British Thoracic Society. Pulmonary disease caused by M. malmoense in HIV negative patients: 5-yr followup of patients receiving standardised treatment. Eur J Respir J 2003; 21: $478-482$

${ }^{15}$ Schäfer H, Ewig S, Hasper E et al. Bronchopulmonary infection with Mycobacterium malmoense presenting as a bronchoesophageal fistula. Tubercle \& Lung Dis 1996; 77: $287-290$

${ }^{16}$ Schaubschläger WW, Schröder KH, Mauch H et al. Atypische Mykobakteriosen: Erkrankungen mit Mycobacterium malmoense. Pneumologie 1990; 44: 699-703

${ }^{17}$ Schmoor P et al. Infection cutanée à Mycobacterium malmoense chez une malade immunocompétente. Ann Dermatol Venereol 2001; 128: $139-140$

18 Syed AA, O'Flanagan J. Mycobacterium malmoense infection. An unusual cause of wrist swelling. J Hand Surg 1998; 23 B: $811-812$

${ }^{19}$ Tortoli E et al. Mycobacterium malmoense in Italy: the modern Norman invasion? Eur J Epidemiol 1997; 13: 341 - 346

${ }^{20}$ White VLC, Al-Shahi R, Gamble E et al. Transverse myelopathy and radiculomyelopathy associated with pulmonary atypical Mycobacterium infections. Thorax 2001; 56: $158-160$

${ }^{21}$ Whitehead SE, Allen KD, Abernethy VE et al. Mycobacterium malmoense septic arthritis. J Infect 2003; 46: 60-71

22 Zaugg M, Salfinger M, Opravil M et al. Extrapulmonary and disseminated infections due to Mycobacterium malmoense: case report and review. Clin Infect Dis 1993; 16: 540-549 